DOI: http://dx.doi.org/10.11606/issn.1984-4867.v27i2p342-363

\title{
O Planejamento Estratégico para Implantação da Sustentabilidade na Copa do Mundo FIFA 2014 no Brasil
}

\section{Strategic Planning for Sustainability Implementation in the FIFA World Cup 2014 in Brazil \\ Planificación Estratégica para la Aplicación de Sostenibilidad en la Copa Mundial de la FIFA 2014 en Brasil}

Elaine Gomes Borges da Silva ${ }^{1}$

\begin{abstract}
Resumo
Esta pesquisa descritiva traz a análise documental do planejamento estratégico para a aplicação do conceito de sustentabilidade na Copa do Mundo FIFA 2014. Por meio deste estudo descobriu-se que o evento deveria estar em consonância com as políticas públicas do governo federal vigentes à época de 2012, além de primar pelos aspectos ambientais na execução das obras das instalações esportivas e seu entorno. Verificou-se que para que o evento estivesse em concordância às recomendações e requerimentos da entidade FIFA deveria ser seguido o check-list para obtenção da certificação LEED, ser avaliada a emissão de gases de efeito estufa em complementaridade a outras ações em prol da garantia da qualidade de vida da sociedade e do meio ambiente e que ao final deveria ser publicado um relatório de sustentabilidade daquele evento que deverá ser um paradigma para a sustentabilidade em eventos futuros. As conclusões desse estudo revelam que a dimensão ambiental da sustentabilidade é prioridade para os organizadores desse megaevento.
\end{abstract}

Palavras-chave: Plano estratégico; Sustentabilidade; Copa do Mundo FIFA 2014.

\begin{abstract}
This descriptive research brings the documentary analysis of strategic planning for the implementation of the concept of sustainability in the FIFA World Cup 2014. Through this study it was found that this should be in line with the policies of the federal government force at 2012, besides excelling the environmental aspects in the works of sports facilities and its surroundings. It was found that for the event were in agreement with the recommendations and the FIFA organization requirements should be followed the checklist for obtaining LEED certification, be evaluated green house gases complementary too their actions in support of quality assurance life of society and the environment and that the end would be publishing a sustainability report that event to be a paradigm for sustainability in future events. The findings of this study reveal that the environmental dimension of sustainability is a priority for the organizers of this mega event.
\end{abstract}

Keywords: Strategic plan; Sustainability; FIFA World Cup 2014.

\footnotetext{
${ }^{1}$ Mestra em Turismo e Especialista em Gestão de Negócios Turísticos pela Universidade de Brasília (UNB). Pesquisadora do Projeto "Desenvolvimento Científico do Processo Teórico-Metodológico para Capacitação em Voluntariado Público" do Centro de Apoio ao Desenvolvimento Tecnológico da Universidade de Brasília (UnB). Brasília, Distrito Federal, Brasil. E-mail: elaineborgesturismo@ hotmail.com
} 


\section{Resumen}

Esta investigación descriptiva trae el análisis documental de la planificación estratégica para la práctica del concepto de sostenibilidad en la Copa Mundial de la FIFA 2014. A través de este estudio se encontró que el evento debería estar en consonancia con las políticas del gobierno federal en el momento de 2012, además sobre saliendo los aspectos medio ambientales en las obras de instalaciones deportivas y sus alrededores. Se encontró que para que el evento estuviese de acuerdo con las recomendaciones y los requisitos de la organización de la FIFA deberían seguir la lista de verificación para la obtención de la certificación LEED, evaluarlos gases de efecto invernadero complementarias de otras acciones en apoyo de la garantía de calidad la vida de la sociedad y el medio ambiente y que al final se publicará un informe de sostenibilidad del evento a ser un paradigma de la sostenibilidad para los eventos futuros. Los resultados de este estudio revelan que la dimensión ambiental de la sostenibilidad es una prioridad para los organizadores de este mega evento.

Palabras clave: Plan estratégico; Sostenibilidad; Copa Mundial de la FIFA 2014.

\section{Introdução}

Uma característica da Copa do Mundo realizada de 12 de junho a 13 de julho de 2014, em 12 cidades sedes brasileiras, foi a Estratégia de Sustentabilidade a ser adotada em suas fases de organização e na execução do projeto de intervenção para as obras nas instalações esportivas.

Na década de 1990, refletindo as discussões mundiais que culminaram no conceito de sustentabilidade e desenvolvimento sustentável de 1987, surgiram as primeiras tentativas de aplicar esse novo paradigma aos megaeventos esportivos. (MASCARENHAS, BIENESTEIN e SÁNCHEZ, 2011). E em 1997, foi criada a Declaração Ostersund do Turismo de Megaeventos que recomenda maior compreensão sobre: os efeitos desse segmento na criação da imagem dos países, regiões e turismo local; efeitos de curto e longo prazo relacionados a atração de visitantes e as consequências sociais e econômicas por ele originados; a importância para a realização de metas econômicas e sociais essenciais; o desenvolvimento de estratégias para a realização dentro da estrutura geral da política de planejamento turístico local, regional e nacional e as abordagens para otimizar os impactos turísticos de megaeventos recomenda ainda que estes sejam analisados em função dos efeitos sociais, econômicos, ambientais, culturais e físicos; que haja um aperfeiçoamento na coordenação global da administração e pesquisa dos megaeventos, nos padrões e na metodologia de pesquisa que possibilitem comparações globais consistentes entre os eventos e maior cooperação entre 
governos, universidades e setores especializados para auxiliar o desenvolvimento social e econômico originado pelos megaeventos (NIELSEN, 2002).

Este artigo foi elaborado para uma pesquisa sobre o relatório de sustentabilidade publicado pela Federação Internacional de Futebol Associado- FIFA, em dezembro de 2014. Espera-se que este contribua para a compreensão de planejamentos estratégicos que tenham a Sustentabilidade como tema principal, que sejam contempladas todas as suas dimensões em eventos de grande porte. Para responder sobre a estratégia de sustentabilidade e suas dimensões que o plano contemplou, o desempenho e as articulações dos stakeholders envolvidos no projeto de intervenção dos estádios das 12 cidades sedes dos jogos, foi elencado o objetivo geral de analisar a estratégia de sustentabilidade para a copa do Mundo FIFA de 2014 por meio dos documentos: Sustainability Strategy- concept, o Sustainability: Summary of the 2014 FIFA World Cup Brazil Carbon Footprint e o Football Stadiums Technical recommendations and requirements.

Os objetivos específicos foram levantar os documentos pertinentes ao planejamento do evento alinhados ao tema sustentabilidade para a Copa de 2014, identificar os stakeholders envolvidos na fase preparatória do megaevento. Na segunda fase da pesquisa foi verificar o conceito de sustentabilidade e as dimensões que os documentos contemplam e determinar o papel dos principais stakeholders do planejamento estratégico de sustentabilidade para o megaevento.

Além de introdução, método, referencial teórico e conclusões, o artigo apresenta os subtítulos: Responsabilidade Social e Meio Ambiente na Estratégia e Dimensionamento Ambientalista nas Instalações, que relata os três documentos do plano estratégico de sustentabilidade para a Copa 2014 e, Da Plataforma do World Resources Institute ao Compromisso de Publicação de Relatório.

\section{Método}

De abordagem qualitativa, o artigo é uma pesquisa descritiva cujo instrumento utilizado foi a análise documental da estratégia de sustentabilidade para a Copa de 2014 no Brasil. Neste tipo de pesquisa, a finalidade é observar, registrar e analisar os fenômenos ou sistemas técnicos, sem entrar no mérito dos conteúdos. Não pode haver interferência do pesquisador, que deverá apenas descobrir a frequência com que o fenômeno acontece ou como se estrutura e funciona 
um sistema, método, processo ou realidade operacional. Pode ser compreendida como um estudo no qual se realiza a análise das relações entre as variáveis para, posteriormente, determinar os efeitos resultantes (PEROVANO, 2014). As conclusões levam em conta o conjunto de variáveis que podem estar correlacionadas com o objeto da investigação (PARRA FILHO e SANTOS, 2011).

A revisão bibliográfica que aportou o referencial teórico trouxe os conceitos de megaevento, sustentabilidade, planejamento estratégico e stakeholders consagrados na literatura científica. Os documentos para as análises receberam cruzamento de informações com alguns dos demais envolvidos na fase de organização e execução do megaevento esportivo: a Global Report Initiative, o Grupo OAS, a OAS Arenas, o World Resources Institute, o GHG Protocol Control e US Green Building Council que a partir da consulta dos seus planos - missões, visões e códigos de ética e conduta - possibilitou responder questões sobre a inserção do conceito da sustentabilidade naquele evento no Brasil.

\section{Referencial Teórico}

A compreensão dos megaeventos ainda é muito recente, há poucas décadas os eventos esportivos internacionais ganharam a conotação de "mega" (MASCARENHAS, BIENENSTEIN e SÁNCHEZ, 2011). O megaevento tem o mesmo significado do macro evento, ou seja, permite a ocupação de todo o parque hoteleiro de uma ou várias cidades; mobiliza milhares de pessoas nos processos de organização e operação; tem participação do setor público em suas três esferas - federal, estadual e municipal. A coordenação geralmente é estabelecida a encargo de entidade pública - prefeitura, governo estadual ou ministérios federais - que costuma lançar mão da contratação de terceiros para atuar em diversas áreas, inclusive com a participação do setor privado por meio de parcerias; a divulgação se dá a nível internacional; costuma atrair e envolver diversos atores, mobilizando toda a região da sede; e, geralmente para sua promoção, as empresas escolhidas são as maiores do mercado nacional e internacional. A Copa do Mundo da FIFA é um real exemplo desse tipo de evento por sua dimensão, pela data e período fixo, ocorrendo de quatro em quatro anos, e pelo perfil dos participantes (CAMPOS et al., 2000).

A Copa do Mundo é evento internacional, de esporte único (futebol), sua data costuma ser fixada em um mês de duração, ocorre a cada quatro anos em um país distinto, seu público 
compreende o público geral e seus participantes envolve atletas, dirigentes do esporte, chefes de Estado, voluntários, organizadores, trabalhadores e prestadores de serviços, entre outros.

O número de visitantes em um megaevento excede um milhão de pessoas, com orçamento de pelo menos US\$ 500 milhões. É tido como “imperdível”. (ALLEN et al, 2008). Acontecimento único, de duração limitada e atenção em massa, em termos das dimensões físicas ou financeiras, podem atrair atenção por meio de propaganda paga ou da publicidade gratuita devido o crescente interesse midiático (NIELSEN, 2002). E "sua magnitude afeta economias inteiras e repercute na mídia global" (GETZ, 1997, p. 6). Essas ações servem para o aumento da consciência, atração e lucratividade de um destino turístico a curto ou longo prazo cujo sucesso depende de sua originalidade, status ou significado favorável para criar interesse e atrair atenção (RITCHIE e YANGZHOU, 1987).

As interpretações sobre a sustentabilidade tomam como ponto de partida o consenso alcançado pela Comissão Mundial sobre Meio Ambiente e Desenvolvimento - CMMAD em 1987 (UNEP, 2011) como “[...] aquele que atende as necessidades das gerações atuais sem comprometer a capacidade das gerações futuras de atenderem as suas necessidades e aspirações" (ONU - Relatório Brundtland, 1987).

Para Jacobi (2003, p. 196), “a noção de sustentabilidade implica, portanto, uma inter-relação necessária de justiça social, qualidade de vida, equilíbrio ambiental e a ruptura com o atual padrão de desenvolvimento". Por sua vez, Sachs (2004) afirma que desenvolvimento sustentado é norteado no crescimento ampliando o lucro e a igualdade, baseado em ações estratégicas ambientais, territoriais, econômicas, sociais, culturais e políticas.

De acordo com Kotler (1975), planejamento estratégico é uma metodologia gerencial que permite estabelecer a direção a ser seguida pela organização, com o objetivo de maior grau de interação com o ambiente e isto engloba âmbito de atuação, macro políticas, políticas funcionais, filosofia de atuação, macro estratégia, estratégias funcionais, macro objetivos e objetivos funcionais.

Stakeholder inclui aqueles indivíduos, grupos e outras organizações que possuem interesse nas ações de uma empresa e possuem habilidade para influenciá-la (SAVAGE et al, 1991). Segundo Freeman e Mc Vea (2010), a teoria dos stakeholders está sustentada na sociologia, no comportamento organizacional e na política de interesses de grupos específicos, é 
abordagem que prioriza o gerenciamento de relacionamentos entre os diversos atores que compõem o universo empresarial, procurando integrar seus diferentes interesses.

Mitchell et al (1997) estabelecem uma classificação de stakeholders com base nos atributos: poder, legitimidade e urgência. Para estes autores eles podem ser visíveis ou latentes, e podem se manifestar a qualquer momento. Podem ser considerados stakeholders: os acionistas, os fornecedores, os empregados, os administradores, a comunidade e os consumidores. (FREEMAN, 2010); funcionários, governo, mídia e Organizações Não Governamentais ONG's (OLIVEIRA, 2008), os sindicatos e os concorrentes (SOUSA e ALMEIDA, 2006).

\section{Responsabilidade Social e Meio Ambiente na Estratégia e Dimensionamento Ambientalista nas Instalações}

Sustainability Strategy-concept- foi amparada nas experiências adquiridas através dos programas ambientais e de desenvolvimento social em torneios da FIFA desde o ano de 2005, em diretrizes internacionais como o ISO 26000 e a Global Reporting Initiative - GRI, uma organização não governamental internacional que promove a elaboração de relatórios de sustentabilidade, e nas políticas de desenvolvimento do Governo Federal. (FIFA, 2013)

A estratégia forneceu um panorama baseado no conceito estratégico do Conselho Deliberativo de Responsabilidade Social Corporativa - RSC, composto pela FIFA e por representantes do Comitê Organizador Local - COL. Ela mostrava as abordagens de desafios e oportunidades de sustentabilidade para interagir com entidades envolvidas durante a realização do megaevento, visando a redução dos impactos negativos e maximizando os positivos para a sociedade e o meio ambiente. Estas foram acordadas para que a visão da sustentabilidade fosse reconhecida pelas partes interessadas (organizadores e stakeholders), e que estes tomassem medidas para que o evento fosse mais sustentável, inspirassem iniciativas no país sede e no mundo e que seus resultados servissem de modelo para suas próximas edições e demais eventos esportivos. Pela implementação de ações e projetos que colaborassem com os stakeholders na promoção da sustentabilidade alavancando oportunidades, reduzindo riscos e promovendo legado duradouro.

O Planejamento Estratégico e os objetivos foram alicerçados em responsabilidade social e meio ambiente - no programa ambiental a priorização da gestão de resíduos, água, energia, transporte, compras sustentáveis e mudanças climáticas. Sob a promoção e a integração dos 
princípios da ISO 26000 nas gestões e comemoração do evento foi abordado: responsabilização (accountability) - pelas decisões e atividades causadas principalmente pelas consequências negativas mais expressivas com medidas para evitar que se repitam; ética e anticorrupção: agir com valores de honestidade, igualdade e integridade; transparência nas ações que causam impacto, com publicação de políticas, decisões e atividades; respeito consideração, respeito e resposta aos interesses dos stakeholders; Estado de direito: cumprimento dos requisitos legais em todas as jurisdições onde a organização atua, mesmo na ausência de medidas adequadas ao cumprimento de leis e regulamentos; colaboração interação com outras entidades que compartilham os princípios de minimização de impactos negativos, colaboração com as três esferas governamentais (federal, estadual e municipal) assegurando o alinhamento das atividades na busca de metas em comum e maior impacto global; abordagem de longo prazo -priorizar o impacto a longo prazo e iniciativas sustentáveis capazes de deixar legado duradouro; alavancagem de oportunidade - uso da plataforma do megaevento para comunicar e alavancar a conscientização e educação de grande público sobre questões de relevância local e internacional.

Pontos da ISO 26000 consideram que responsabilidade social são práticas legais de cooperação; questões relativas aos consumidores; práticas de trabalho; direitos humanos, envolvimento e; desenvolvimento das comunidades. Estas atividades deveriam ser associadas ao Football for Hope, projeto de promoção de mudanças sociais da FIFA.

A estratégia foi alinhada às diretrizes das políticas públicas da presidência da República no governo em exercício no ano de 2012: expandir e fortalecer a democracia; aumentar o crescimento econômico; implementar um projeto de desenvolvimento em larga escala e a longo prazo; defender o meio ambiente e garantir o desenvolvimento sustentável; erradicar a pobreza e reduzir a desigualdade; melhorar as condições de trabalho e facilitar o acesso de jovens ao emprego formal; garantir educação de qualidade e à formação profissional; transformar o Brasil em potência científica e tecnológica; melhorar o acesso a serviços de saúde de qualidade; suprir as necessidades básicas do cidadão, como moradia, saneamento e transporte; promover a cultura brasileira e o diálogo com outras culturas; garantir a segurança civil e combate ao crime organizado e; defender a soberania nacional por meio de uma presença ativa e altiva do Brasil no mundo.

Para as partes interessadas o desenvolvimento e implementação de programa socioambiental estabeleceria participação constante e construtiva dos principais atores como a FIFA, o COL, 
o governo federal, as sedes, os parceiros comerciais da FIFA, autoridades responsáveis pelos estádios, prestadores de serviços, fornecedores com os outros atores envolvidos (espectadores, ONGs - organizações não governamentais, comunidades locais, Confederação Brasileira de Futebol- CBF e organizações multilaterais de desenvolvimento:

Quadro 1: Stakeholders latentes ou visíveis e responsabilidades

\begin{tabular}{|c|c|}
\hline ENVOLVIDOS & RESPONSABILIDADES \\
\hline FIFA e COL & $\begin{array}{l}\text { - Colaboração com áreas funcionais de modo a integrar } \\
\text { sustentabilidade à gestão e às operações do mundial. }\end{array}$ \\
\hline $\begin{array}{l}\text { Governo Federal, sedes e } \\
\text { autoridades responsáveis pelos } \\
\text { estádios }\end{array}$ & $\begin{array}{l}\text { - Trabalho conjunto do governo federal e Equipe de RSC } \\
\text { através da CTMAS - Câmara Temática de Meio } \\
\text { Ambiente e Sustentabilidade criada pelo ME - } \\
\text { Ministérios dos Esportes. } \\
\text { - Contato com sedes e autoridades responsáveis pelos } \\
\text { estádios para conhecimento de suas iniciativas sociais e } \\
\text { ambientais. }\end{array}$ \\
\hline $\begin{array}{l}\text { Parceiros comerciais } \\
\text { (Adidas, Coca-Cola, Sony, } \\
\text { Visa, Emirates, Hyundai/Kia } \\
\text { Motors) }\end{array}$ & $\begin{array}{l}\text { - Integração através de apoio na forma de produtos e } \\
\text { serviços. } \\
\text { - Alinhamento das iniciativas específicas do programa } \\
\text { ambiental e/ou do programa Football for Hope. } \\
\text { - Busca de colaboração de certos patrocinadores, apoiadores } \\
\text { nacionais e licenciados especialmente com os ativos na } \\
\text { promoção do desenvolvimento social ou proteção } \\
\text { ambiental. }\end{array}$ \\
\hline $\begin{array}{l}\text { Prestadores de serviços } \\
\text { fornecedores }\end{array}$ & $\begin{array}{l}\text { - Promoção de políticas de compras sustentáveis e } \\
\text { integração de critérios de sustentabilidade. }\end{array}$ \\
\hline Stakeholders & $\begin{array}{l}\text { - Apoio a ONGs que utilizam o futebol como ferramenta } \\
\text { para mudanças sociais, por meio do programa Football } \\
\text { for Hope. }\end{array}$ \\
\hline
\end{tabular}

Fonte: Elaborado pela autora com informações da Estratégia de Sustentabilidade

Consta neste que, pós evento seria publicado um abrangente relatório de sustentabilidade desenvolvido pelo Global Reporting Initiative - GRI. Conforme classificam os autores Freeman (2010), Oliveira (2008) e Sousa e Almeida (2006), todos os envolvidos relacionados no quadro acima são considerados stakeholders, estejam a desempenhar o papel de acionistas, fornecedores, empregados, administradores, comunidade, consumidores, funcionários, governo, mídia, Organizações Não Governamentais, sindicatos e a concorrência, tenham eles relação de poder, legitimidade ou urgência como estabelece Mitchell, Agle e Wood (1997).

Sustainability: Summary of the 2014 FIFA World Cup Brazil Carbon Footprint- apresentado por ocasião da Rio+20 - Conferência das Nações Unidas sobre Desenvolvimento Sustentável, relatou as diretrizes das políticas e/ou legislação do país anfitrião para trabalho conjunto com 
a detentora do megaevento de forma sustentável. É um norteador acerca do comprometimento da entidade FIFA e do COL, um guia para alcançar a missão da organizadora FIFA: "A construção de um futuro melhor".

Este foi elaborado em decorrência de metas de proteção ambiental presentes nas Copas do Mundo da Alemanha e África do Sul e que passaram a ser item obrigatório para futuros acordos de candidatura a fim de evitar, reduzir e compensar os impactos ambientais negativos do evento (CSR FIFA, 2013). Cumprindo com os princípios de contabilização e comunicação do Greenhouse Gas Protocol - GHG Protocol e da norma ISO 14064.1, os fundamentos da Estratégia de Sustentabilidade e os principais fundamentos de orientação metodológica da pegada de carbono foram: a produção de um relatório completo, relevante, consistente, preciso e transparente; a contabilização das emissões diretas e indiretas dos escopos 1, 2 e 3 das fases do evento; a reportagem ao máximo possível das emissões de todos os gases de efeito estufa relacionados pelo Protocolo de Kyoto; a definição dos limites do projeto de maneira ampla, para incorporar as emissões sob controle operacional da FIFA e do COL; a definição dos critérios claros de inclusão e exclusão para a tomada de decisões sobre o que deveria conter no escopo; a identificação de um conjunto de fatores de emissão de carbono, dentro do possível representativo da localização e da configuração do evento; e, fornecimento das informações necessárias para a concepção de estratégias de mitigação e compensação de emissões de Gases de Efeito Estufa - GEE.

Seja para uma organização ou um evento, a definição dos limites é uma atividade crítica para assegurar que uma pegada de carbono seja uma representação real das emissões de GEE, e para o cumprimento de alguns dos princípios de contabilização e comunicação do GHG Protocol. Todavia, reconhecendo que ele foi concebido para a contabilização das emissões corporativas, seria necessária a adaptação para aplicar o Protocolo em um evento esportivo.

A minimização dos impactos negativos na esfera ambiental deveriam estar presentes na fase organizacional do megaevento: Preparatória, Realização da Copa das Confederações e a Realização da Copa do Mundo da FIFA. Os limites organizacionais para a pegada de carbono são definidos a partir das unidades também organizacionais necessárias para preparar e realizar o evento. Segundo consta em GHG Protocol (2014) uma empresa tem o controle financeiro sobre a operação se ela tiver a capacidade de dirigir suas políticas financeiras e operacionais, visando obter vantagens econômicas com suas atividades. Considera-se a existência de controle operacional quando, apesar de não existir o controle financeiro, uma 
empresa tem autoridade plena a introdução e implementação de suas políticas operacionais naquela ação.

O GHG Protocol (2014) tem duas abordagens principais para a definição de limites organizacionais, são elas a participação no capital e o controle operacional. Para a Copa do Mundo da FIFA Brasil 2014, foi escolhida a abordagem de controle operacional para permitir a alocação de emissões de acordo com o nível de controle de uma empresa, proporcionando uma ação mais relevante e completa. Como resultado, eventos, atividades preparatórias e de realização sobre os quais a FIFA e o COL possuíam controle operacional ficaram dentro dos limites organizacionais da pegada de carbono.

Quanto aos limites operacionais, estes referiam-se a uma etapa que tinha por objetivo identificar as fontes de emissões no âmbito das operações controladas. Essa tarefa exigia uma compreensão ampla das operações e envolvimento do pessoal da FIFA e do COL nos diferentes níveis organizacionais. Tal compreensão foi necessária para assegurar que as fontes de emissão fossem plenamente identificadas, bem como para garantir que fossem devidamente categorizadas como diretas ou indiretas. Naquela época, ainda não haviam sido confirmados o local e a data de realização das fan fests internacionais. As estimativas foram baseadas em eventos anteriores realizados pela FIFA.

O Período de contabilização era necessário para haver consistência com os limites de projetos estabelecidos para o megaevento que foi estipulado de 2011 - sorteio preliminar da Copa do Mundo da FIFA Brasil 2014 - até o fim de setembro (época de devolução dos estádios/arenas aos seus entes responsáveis). Em linhas gerais, o processo de decisão propôs que uma fonte de emissões poderia exercer influência direta caso fosse considerado crítico pelas principais partes interessadas. Os critérios de inclusão/exclusão discutidos na metodologia do documento orientavam para a determinação clara das emissões que deviam estar contidas no escopo do projeto sobre a pegada de carbono, considerando a proporção global do megaevento, sua magnitude, complexidade, e as múltiplas interfaces que apresentou com outras iniciativas do governo brasileiro e dos governos das cidades sede, exigindo o estabelecimento de conjunto de critérios claros para possibilitar dar suporte às ações.

Alguns conceitos-chave associados à interpretação do processo decisório são a consequência direta - emissões resultantes dos eventos preparatórios e dos torneios e o controle operacional - uma das abordagens de consolidação registradas no GHG Protocol foi o estabelecimento do 
limite. A FIFA e o COL teriam o controle operacional quando possuíssem autoridade total para introduzir e implementar suas próprias práticas e políticas operacionais. A influência da FIFA e do COL nos processos decisórios poderiam impactar diretamente as emissões associadas aos eventos. As informações seriam consideradas materiais se, por sua inclusão ou exclusão observasse influência em quaisquer decisões ou ações tomadas pelos seus usuários. Uma discrepância material é um erro, seja por equívoco, omissão ou erro de cálculo que resulta em uma quantidade reportada ou declarada diferente do valor ou significado real. As emissões relacionadas às principais atividades operacionais e à missão da FIFA e do COL, bem como as emissões consideradas importantes pelas principais partes interessadas ou pela percepção do público, são consideradas relevantes independentemente da sua materialidade (GHG PROTOCOL, 2014).

Football Stadiums - Technical recommendations and requirements- direcionado para as intervenções dentro da instalação esportiva.

Para abrigar seus participantes, suportar a carga, disponibilizar o acesso e a mobilidade, ou seja, as qualificações para sediar o megaevento esportivo, os estádios brasileiros em suas 12 cidades sede passaram por obras que seguiram o documento que sistematizava como devem ser realizadas as readequações aos padrões técnicos da FIFA. Este documento FIFA (2007) abrange as recomendações e requerimentos dividindo nas áreas de: Decisões pré-construção, Segurança; Orientação e estacionamento; Campo; Jogadores e partidas oficiais; Espectadores; Hospitalidade; Mídia; Fornecimento de luz e energia; Comunicações e áreas adicionais. Para a Copa 2014 foi lançada sua versão em português, a 5ª edição FIFA (2011).

Neste documento consta o conceito de sustentabilidade para a FIFA que está explicitada na dimensão ambiental através do programa Green Goal (Gol Verde). As principais metas daquele programa são: reduzir o consumo de água potável, evitar e/ou reduzir a emissão de resíduos, criar sistemas de abastecimento de energia mais eficientes e aumentar o uso do transporte público nos eventos FIFA. Esse programa começou durante os preparativos para a Copa do Mundo FIFA 2006 na Alemanha e, considerando não existir metodologia padronizada para calcular as emissões de gases de efeito estufa associadas a eventos esportivos de grande porte, este estudo de pegada de carbono baseou-se principalmente no GHG Protocol, e incorporou orientações técnicas adicionais da norma ISO 14064.1. Além disso, o estudo foi baseado em resultados introduzidos tanto pela Copa do Mundo da FIFA 2010 na África do Sul como pelos Jogos Olímpicos de Londres 2012. 
Por meio do programa Green Goal (Gol Verde) propôs investimentos relacionados ao uso da água, a remoção dos rejeitos, a atividade de baixo consumo de energia e obtenção de certificação na categoria de Construção Sustentável e determinação de pegada de carbono:

- Água - O uso mais responsável de água potável para fins de irrigação deve ser avaliado. O armazenamento de água pluvial para uso no ciclo da água. Um maior potencial econômico pode ser atingido através da instalação de tecnologia de economia de água em instalações sanitárias durante a fase de construção.

- Rejeitos - A remoção de rejeitos representa um elevado custo administrativo de um estádio. Para limitar a quantidade de rejeitos gerados, a reutilização de recipientes de bebidas, reciclando-os através de coleta seletiva, e a introdução de alimentos e produtos promocionais sem embalagens.

- Energia - O projeto de construção de estádios deve empregar atividades de baixo consumo de energia. As áreas com potencial de economia de energia incluem o uso de tecnologia fotovoltaica.

- Certificação - Alegando que a agenda de meio ambiente e sustentabilidade tem se desenvolvido intensamente em todo o mundo, o surgimento de vários métodos e sistemas de certificação tem facilitado o processo de definição de objetivos e a avaliação de impactos.

A Certificação Leadership in Energy Efficient Design - LEED, Método de Avaliação da Sustentabilidade de Edifícios (Building Research Establishment Environmental Assessment Method, BREEAM), seu selo desenvolvido em 2002 pelo US Green Building CouncilUSGBC (Conselho de Construção Sustentável dos EUA) é considerado o principal selo para edificações no mundo (utilizado em 143 países) - objetiva incentivar a transformação dos projetos, obra e operação das edificações, com foco na sustentabilidade de suas atuações. O nível do selo pode variar nas escalas: Certificado (40 - 49), Prata (50-59), Ouro (60-79) e Platina (80 - 110) (US GREEN BUILDING COUNCIL, 2014).

No documento do programa brasileiro, o GHG Protocol defende-se que seus benefícios abrangem o valor econômico com a diminuição dos custos operacionais; diminuição dos riscos regulatórios; valorização do imóvel para revenda ou arrendamento; aumento na velocidade de ocupação; aumento da retenção; modernização e menor obsolescência da edificação; pelo valor social, melhora na segurança e priorização da saúde dos trabalhadores e ocupantes; inclusão social e aumento do senso de comunidade; capacitação profissional; 
conscientização de trabalhadores e usuários; aumento da produtividade do funcionário; melhora na recuperação de pacientes (em Hospitais); melhora no desempenho de alunos (em Escolas); aumento no ímpeto de compra de consumidores (em Comércios); incentivo a fornecedores com maiores responsabilidades socioambientais; aumento da satisfação e bem estar dos usuários; estímulo a políticas públicas de fomento a construção sustentável e o valor ambiental; uso racional e redução da extração dos recursos naturais; redução do consumo de água e energia; implantação consciente e ordenada; mitigação dos efeitos das mudanças climáticas; uso de materiais e tecnologias de baixo impacto ambiental; e, redução, tratamento e reuso dos resíduos da construção e da operação. A certificação avalia as edificações nas categorias (GHG PROTOCOL, 2014):

- Sustainable sites (locais sustentáveis) - refere à seleção de locais e como o desenvolvimento se integra ao meio ambiente natural, ao ambiente social e à rede de transporte público existente. Encoraja estratégias que minimizam o impacto no ecossistema durante a implantação da edificação e aborda questões fundamentais de grandes centros urbanos, como redução do uso de carros e das ilhas de calor.

- Water efficiency (eficiência do uso de água) - objetiva a redução do consumo de água e o uso de fontes alternativas, como a coleta da água pluvial. Promove inovações para o uso racional da água, com foco na redução do consumo de água potável e alternativas de tratamento e reuso dos recursos.

- Energy \& atmosphere (energia e atmosfera) - encoraja o uso de fontes alternativas e trata de formas de redução de emissão de gases do efeito estufa. Promove eficiência energética nas edificações por meio de estratégias simples e inovadoras, como por exemplo simulações energéticas, medições, comissionamento e utilização de equipamentos e sistemas eficientes.

- Materials \& resources (recursos naturais e materiais) - pontua o uso de materiais de construção sustentáveis, materiais com baixa emissão ou emissão zero e materiais de fontes locais. Encoraja o uso de materiais de baixo impacto ambiental (reciclados, regionais, recicláveis, de reuso, etc.) e reduz a geração de resíduos, além de promover o descarte consciente, desviando o volume de resíduos gerados dos aterros sanitários.

- Indoor environmental quality (qualidade de ambiente interno) - trata das questões de criação de um ambiente interno limpo e saudável que utilize ventilação natural e luz solar. Promove a qualidade ambiental interna do ar, essencial para ambientes com alta permanência 
de pessoas, com foco na escolha de materiais com baixa emissão de compostos orgânicos voláteis, controle de sistemas, conforto térmico e priorização de espaços com vista externa e luz natural.

- Innovation in design or innovation in operations (inovação em projeto ou operações) premia soluções inovadoras que auxiliem a redução do impacto da obra no ambiente. Incentiva a busca de conhecimento sobre Green Buildings, assim como a criação de medidas de projetos não descritas nas categorias do LEED.

- Regional priority credits - incentiva os créditos definidos como prioridade regional para cada país, de acordo com as diferenças ambientais, sociais e econômicas existentes em cada local.

No Brasil, o selo já contemplou 90 edificações e coloca-o na posição de quarto país com maior número de registros no ranking mundial.

\section{Da plataforma do WRI Resources Institute ao Compromisso de Publicação de Relatório}

Sobre o GHG Control, sabe-se que esta plataforma originalmente desenvolvida nos Estados Unidos no ano de 1998, pelo World Resources Institute- WRI, é uma ferramenta utilizada para entender, quantificar e gerenciar emissões de GEE. Atualmente é o método mais usado pelas empresas e governos para a realização de inventários e, dentre de suas características, destacam-se por oferecer uma estrutura para contabilização de GEE, por seu caráter modular e flexível, a neutralidade em termos de políticas ou programas e ser baseada em um amplo processo de consulta pública. (GHG PROTOCOL, 2014)

No ano de 2008, esse método foi adaptado ao contexto nacional no Programa Brasileiro GHG Protocolem parceria da WRI com o Gvces - Centro de Estudos da Sustentabilidade da Fundação Getúlio Vargas, o MMA - Ministério do Meio Ambiente, o CEBDS - Conselho Empresarial Brasileiro para o Desenvolvimento Sustentável, com o WBSCD - World Business Council for Sustainable Development e 27 empresas fundadoras. (GHG PROTOCOL, 2014)

Conforme a plataforma de certificação LEED, as implicações de custo para a construção de um estádio sustentável variam e quanto maior a pontuação atingida pelo projeto, maior é o custo do estádio. FIFA (2011) recomenda aos engenheiros de sistemas e outros profissionais 
que avaliem a adição de equipamentos tais como ar condicionado de baixo consumo de energia, iluminação de LED e comissionamento do sistema, que prevejam também que estes sistemas podem reduzir o consumo de energia e os custos de manutenção ao longo da vida útil do estádio. Para o planejamento geral do projeto, instruem a estes profissionais que atentem para o orçamento de um estádio bem projetado que considere o custo de energia e o operacional para um inter-relacionamento custo/benefício.

De acordo com FIFA (2011), os benefícios das construções sustentáveis incluem aspectos econômicos, sociais e ambientais. Os econômicos seriam: redução dos custos operacionais; aumento do valor do ativo e dos lucros; aumento da produtividade e satisfação dos funcionários; otimização do desempenho econômico durante o ciclo de vida. Na esfera social os itens elencados foram: a saúde das comunidades na melhora do ar; das condições térmicas e da acústica; melhora do conforto e da saúde dos ocupantes; minimização da pressão sobre infraestrutura local; contribuição para a melhoria da qualidade de vida em geral. No âmbito ambiental estava previsto: melhora e proteção de ecossistemas e da biodiversidade; melhora da qualidade do ar e da água; redução da geração de resíduos sólidos; conservação dos recursos naturais; e, redução das emissões de carbono.

Os princípios da construção sustentável de acordo com a FIFA (2011, p.39) incorpora práticas de projeto, construção e operações que reduzem ou eliminam significativamente o impacto negativo sobre o meio ambiente e seus ocupantes. A construção sustentável permite a utilização eficiente de recursos e contribui para mitigar as alterações climáticas, criando ambientes residenciais e de trabalho mais saudáveis e produtivos.

Na concepção da responsável pelas obras de acabamento das instalações esportivas, a empresa OAS Engenharia/Arenas, fragmento do GRUPO OAS, na atualidade as arenas multiuso representam verdadeiros marcos de desenvolvimento socioeconômico para os clubes que abrigam, os municípios que as recebem, as comunidades que as adotam e para as marcas que as patrocinam. Seus empreendimentos visam atender de forma harmônica as expectativas de cada um desses que a OAS Arenas identifica como os pilares do negócio, contemplando aspectos sociais, ambientais, econômicos e promocionais envolvidos no processo (OAS ARENAS, 2014)

Conforme reportado em OAS Arenas (2014). Para os clubes é oportunidade de modernizar o próprio estádio, que pode gerar aumento de receitas e criação de diferencial competitivo. $\mathrm{O}$ 
desafio é o não comprometimento de recursos que venham a colocar em risco o ativo principal do clube, o futebol profissional. Para as cidades significaria ganhos sociais e econômicos gerados a partir da implantação, implementação e gestão correta de uma arena multiuso independentemente da realização de megaeventos. Destacam-se alguns benefícios para o centro urbano, o município ou determinada comunidade entre eles seria a atração de empresas e retenção de mão de obra local.

A ampliação do potencial turístico com a boa gestão de uma arena multiuso, com um calendário de eventos esportivos, artísticos e até mesmo religioso bem estruturado, tem o poder de atrair público de fora dos limites da cidade, trazendo receita adicional ao município, não só advinda da venda de ingressos, como também de hospedagem, alimentação, transporte e demais itens relacionados ao turismo. (OAS ARENAS, 2014)

O processo de planejamento, construção e gestão de uma arena multiuso exige uma série de competências que nem sempre estão disponíveis. As arenas permitem a reconfiguração das cidades, criando novas áreas de desenvolvimento pela valorização gerada por estes projetos associados a minucioso planejamento de todo o seu complexo viário e imobiliário. Funcionando como estímulo para o crescimento e vitalidade econômica de toda uma ampla área a sua volta, pode revitalizar bairros inteiros com a implantação de hotéis, restaurantes, bares, lojas, shopping centers, centros culturais e edifícios de escritórios. (OAS ARENAS, 2014)

Conforme a GRI, com o avanço da revolução digital, as mídias tradicionais se tornam menos efetivas para a construção e divulgação das marcas. Assim, tem-se a busca por novos meios de expressão para resgatar a atenção do público e agregar significado às marcas. Nesse cenário, o apoio ou patrocínio a empreendimentos de interesse público e eventos de grande mobilização popular surge como uma das mais interessantes e efetivas mídias do século XXI, unindo ampla exposição com associação positiva. Concessões e comercializações namingrights, pouring rights, patrocínio de setores, camarotes e outros ativos de arenas multiuso surgem como meio de manutenção de seu valor de mercado e força de comunicação com seus públicos.

Sobre os Relatórios de Sustentabilidade, segundo a GRI (2012), o motivo inicial que desencadeia uma organização querer publicar um relatório está relacionado à crença que este a beneficiará de alguma forma, com fatores motivacionais que podem ser: internos ou externos, tangíveis ou intangíveis, financeiros ou morais, demonstração de compromisso e 
transparência. E as razões pelas quais uma empresa decide relatar pode variar ao longo dos anos. Na última década, o número de organizações e indivíduos que questionam as empresas sobre seu desempenho social e ambiental alavancou: investidores, clientes, funcionários, moradores das comunidades e ativistas tem expressado suas preocupações e questionamentos sobre um compromisso empresarial com conduta responsável.

Conforme documentado em GRI (2012) empresas se utilizam de indicadores de gestão, isto é, uma ferramenta para compreender sua contribuição na solução dos problemas atuais da sociedade e apresentar seu negócio a partir de uma nova perspectiva mais bem posicionada num contexto empresarial futuro como parte importante dos processos decisórios e de inovação, estar pronta para seguir a legislação vigente ou futura e a necessidade de demonstrar capacidade de participar em mercados competitivos, planejar atividades, tornar-se mais sustentável e posicionar a empresa.

De acordo com GRI(2012) para as organizações que já preparam este tipo de relatório, a comunicação com os stakeholders e com a sociedade é frequentemente citada como o motivo primário para começar a relatar que esse processo melhorará a credibilidade e a reputação perante os investidores, clientes e membros da comunidade. Muitas organizações percebem que, embora as agências reguladoras concedam às empresas licenças de operação, as organizações também precisam obter da sociedade ou manter uma espécie de licença social de operação.

GRI (2012) aponta que em vários países e jurisdições, os relatórios socioambientais já são uma exigência da legislação que empresas de certo porte ou com ações na bolsa de valores, por exemplo, incluam informações ambientais e/ou sociais em seus relatórios anuais. Alguns já exigem por escrito código de ética adotado por seu presidente, diretor financeiro e diretor de contabilidade.

Provavelmente razões que levam ao reconhecimento da relevância em publicar esses relatórios são a complexidade das questões econômicas, ambientais e sociais que estão se tornando cada vez mais do interesse e consciência da sociedade e que organizações com alto desempenho - por meio de processos geralmente com recursos limitados - têm que rapidamente tomar decisões, podendo facilmente levar a cometer erros e impactar negativamente em várias áreas. (GRI, 2012) 
Conforme a GRI (2012), organizações empresariais ou sem fins lucrativos com alto desempenho ambiental, maior redução de emissões e que publicam relatórios de sustentabilidade costumam ser mais respeitadas e liderar nos seus setores, que um relatório de sustentabilidade melhora a impressão sobre a empresa, leva a demanda pela compra de seus produtos e/ou falar bem da empresa para terceiros. Seus defensores sustentam a ideia que este proporciona credibilidade, comparabilidade, abrangência, maior equilíbrio e legitimidade. Já seus críticos defendem que a obrigatoriedade em relatar é pouco flexível e que não estimula a inovação ou transparência para uma questão complexa e em constante mudança como a sustentabilidade.

$\mathrm{Na}$ atualidade, a capacidade de uma empresa de todos os portes e setores relatar as questões de suas operações com confiabilidade e coerência, tem um valor muito alto no contexto atual em que as conexões digitais permitem notícias instantaneamente e rapidamente propagadas, é esperado que organizações com alto desempenho monitorem seus dados de caráter positivo ou não em várias áreas. As organizações devem lidar com seus pontos fracos e os pontos fortes a fim de mais que maximizar seus impactos positivos, compensar e prestar contas dos danos causados por suas atividades com transparência, profissionalismo e responsabilidade.

Contudo é sabido que para uma marca permanecer no mercado competitivo e posicionar-se a frente da concorrência terá que buscar sempre por um diferencial, vantagem ou revolucionar em seu ramo, a sustentabilidade aparece como modo de alavancagem do negócio e de publicidade das ações muitas das vezes já determinadas pela lei. Deste modo, contabiliza-se com as boas ações em prol do meio ambiente e da qualidade da vida da sociedade que também revertem em capital financeiro para esses empreendimentos.

\section{Conclusões}

Conforme os documentos da FIFA elucidam, este megaevento certamente tem impactos consideráveis sobre a sociedade e o meio ambiente e um plano estratégico com a sustentabilidade como tema principal visaria reduzir impactos negativos e maximizar os efeitos positivos. Foram dois anos de organização e deveriam ser abordadas e executadas ações que garantissem a sustentabilidade aos moldes da FIFA e das premissas das políticas públicas vigentes à época no Brasil. Seriam estádios verdes com manejo de resíduos, uso 
consciente da água, apoio à comunidade, redução e compensação das emissões de carbono, energias renováveis, alguns dos temas fundamentais.

Considera-se não apenas o fato de estar documentado na estratégia, mas sobretudo pelo que apontam os autores que estudam estes eventos de porte macro: a magnitude física e financeira; os interesses e efeitos midiáticos; a publicidade e promoção a nível internacional; a infraestrutura e os equipamentos necessários; a quantidade de participantes e o número de visitantes; os custos do evento; o envolvimento do setor público; a ocupação do espaço (parque hoteleiro) da cidade e seu entorno; a mobilização de pessoas para a organização e operação; parcerias com o setor privado; a contratação de mão de obra e prestadores de serviços.

Segundo informações contidas no sítio eletrônico da FIFA, a entidade previu investir aproximadamente US\$ 20 milhões para a implementação da Estratégia de Sustentabilidade que serviria de paradigma para outros eventos. Os stakeholders, parceiros comerciais da FIFA e outras partes envolvidas também deveriam apoio as iniciativas de sustentabilidade.

As dimensões econômicas, sociais e ambientais da sustentabilidade deveriam ser contempladas. Na fase organizacional, período de execução das obras dos estádios há a priorização da dimensão ambiental da sustentabilidade no evento internacional, explícito no documento Football Stadiums - Recommendations and Requirements. Verificou-se que assim como a estratégia de sustentabilidade para o Brasil foi aprimorada considerando paradigmas de cidades sedes anteriores, ela também foi citada como uma importante plataforma no processo de construção da base para torneios mais sustentáveis. Para a entidade FIFA foi oportunidade de alinhar a estratégia do seu empreendimento Copa do Mundo ao paradigma de desenvolvimento sustentável de um país que tem buscado a expertise em construções sustentáveis, o quarto colocado nesse tipo de edificação.

Outro aspecto é a inclusão das emissões dos eventos ligados à Copa do Mundo da FIFA Brasil 2014, à Copa das Confederações da FIFA Brasil 2013 e aos principais eventos preparatórios que utilizou conjunto próprio de limites organizacionais e operacionais e de períodos de contabilização, avançando para a criação de um único marco de referência para as próximas edições do mundial e outros eventos esportivos. A proposta para a pegada de carbono era de metodologia que incorporasse conceitos básicos de protocolos reconhecidos de contabilização e registro de emissões de GEE, baseado nas principais conclusões de estudos anteriores. 
Sobre os stakeholders, todos deveriam atuar de forma responsável, identificando e prevenindo riscos ambientais no curso de suas atividades, informando imediatamente as instâncias cabíveis dentro de suas respectivas organizações e as autoridades públicas. Enfim, deveria ser evitado de antemão qualquer sinistro que pudesse causar danos. O zelo, uso responsável dos recursos naturais e o incentivo as partes envolvidas na busca por soluções sustentáveis para as suas atividades para a mitigação do impacto.

No que tange a responsabilidade social e promoção do desenvolvimento sustentável, defendem-se o respeito aos direitos humanos e leis vigentes no país e o fomento a inclusão de trabalhadores nos processos do evento, compromisso de responsabilidade, reputação e respeitabilidade. Posto tudo que foi descrito nesta pesquisa, pelos documentos analisados, os principais envolvidos, suas responsabilidades, papéis e a complexidade que envolve a realização de um evento deste no país, caberia levar adiante os estudos do planejamento estratégico de megaeventos com respostas a uma reflexão se esta estratégia de sustentabilidade é uma oportunidade de desenvolvimento sustentável para todo aquele que é um stakeholder seja ele na figura de um indivíduo, um grupo ou organizações que possuem diferentes interesses e habilidade para influenciar com atributos de poder, legitimidade e urgência ou é uma oportunidade de melhora do capital desses visíveis ou latentes stakeholders, fazendo uso do conceito e dos indicadores de sustentabilidade como meio de apresentar seu negócio a partir de uma nova perspectiva para participar em mercados competitivos e posicionar-se a frente da concorrência.

Para melhor compreensão se foram cumpridas as normas demandadas - dentro da metodologia contidas no check-list para a certificação LEED, o Programa Green Goal e a planejamento estratégico - há a necessidade de um estudo que abranja estes documentos descritos neste artigo e o relatório publicado pós evento.

\section{Referências}

ALLEN, Johnny; O’TOOLE, William; MCDONNEL, Ian; HARRIS, Robert. Organização e Gestão de eventos. Rio de Janeiro: Campus, 2008.

Associação Brasileira de Normas Técnicas. ABNT NBR ISSO 14064 - Gases de efeito estufa. Parte 1: Especificação e orientação a organizações para quantificação e elaboração de relatórios de emissões e remoções de gases de efeito estufa. Rio de Janeiro, 2007. 
ABNT NBR IS0 14064 - Gases de efeito estufa. Parte 2: Especificação e orientação a projetos para quantificação, monitoramento e elaboração de relatórios das reduções de emissões ou da melhoria das remoções de gases e efeito estufa. Rio de Janeiro, 2007.

ABNT NBR ISO 14064 - Gases de efeito estufa. Parte 3: Especificação e orientação para a validação e verificação de declarações relativas a gases de efeito estufa. Rio de Janeiro, 2007.

BRUNDTLAND, Gro Harlem. Comissão Mundial sobre Meio Ambiente e Desenvolvimento: o nosso futuro comum.Rio de Janeiro: Fundação Getúlio Vargas. 1991.

CAMPOS, Luiz Claudio de A. Menescal; WYSE, Nely; ARAÚJO, Maria Luiza Motta da Silva. Eventos: oportunidade de novos negócios. Rio de Janeiro: SENAC Nacional, 2000.

FIFA. Football Stadium: recomendation and requirements.2007. Disponível em <http://www.fifa.com/> Acesso em : 02 abr. 2013.

FIFA. Football Stadium: recommendation and requirements. 2011.Disponível em <http://www.fifa.com/> Acesso em : 02 abr. 2013.

FIFA. Sustainability Strategy - Concept. 2012.Disponível em $<$ http://resources.fifa.com/mm/document/fifaworldcup/generic/02/11/18/55/sustainabilitystrategyconc ept_neutral.pdf> Acesso em : 24 out.2013.

FIFA CSR. Responsabilidade Social.2013.Disponível em <http://www.fifa.com/aboutfifa/socialresponsibility/environmental.html>. Acesso em: 16 ago.2014.

FREEMAN, Edward. A Stakeholder theory of the Modern Corporation. Disponível em <http://ufolio.uoregon.edu/lcbleadership/files/2008/03/edward-freeman.pdf>Acesso em : 29 mai.2010.

FREEMAN, Edward; MC Vea, John A. Stakeholder approach to strategic management. Disponível em <http://papers.ssrn.com/sol3/papers.cfm?abstract_id=263511>. Acesso em: 02 abr.2010.

GETZ, Donald. Event Management and Event tourism. New York: Cognizant Communication Corp, 1997.

Global Report Initiative. Relatórios de sustentabilidade da GRI: quanto vale essa jornada?; 2012. Disponível em<https://www.globalreporting.org/resourcelibrary/Portuquese-Starting-Points-2G3.1.pdf> Acesso em: 20 out.2014.

GREENHOUSE GAS PROTOCOL. 2014. Disponível em<http://www.ghgprotocol.org/> Acesso em: 20 out.2014.

GRUPO OAS Disponível em <http://www.oasempreendimentos.com/grupo-oas/>Acesso em: 16 ago.2014.

JACOBI, Pedro. Cadernos de Pesquisa, n. 118, março/ 2003 Cadernos de Pesquisa, n. 118, p. 189-205, março/ 2003.

KOTLER, Philip. Administração de marketing. São Paulo: Atlas, 1975.

MASCARENHAS, Gilmar; BIENENSTEIN, Glauco; SÁNCHEZ, Fernanda. O Jogo continua: megaeventos esportivos e cidades. Rio de Janeiro: UERJ. 2011.

MITCHELL, Ronald K.; AGLE, Bradley R.; WOOD, Donna J. Toward a Theory of Stakeholder Identification and Salience: Defining the Principle of Who and What Really Counts. The Academy of Management Review, Vol. 22, No. 4. Oct., pp. 853-886. 1997.

NIELSEN, C. Turismo e Mídia. O Papel da Comunicação na Atividade Turística. São Paulo: Contexto. 2002.

OAS Arenas Disponível em <http://www.oas.com/oas-com/home.htm> Acesso em: 16 ago.2014.

OLIVEIRA, José A. Puppim de. Empresas na sociedade: sustentabilidade e responsabilidade social. Rio de Janeiro: Elsevier, 2008. 
PARRA FILHO, Domingos. SANTOS, João Almeida. Metodologia Científica. 6. ed. São Paulo: Futura, 1998.

PEROVANO, Dalton Gean. Manual de Metodologia Científica. Paraná: Juruá, 2014.

RITCHIE J. R. B., YANGZHOU J. The Role \& Impact of Mega-events and Attractions on National and Regional Tourism Development: A Conceptual and Methodological Overview. In: Proceedings of the 37th Annual Congress of the International Association of Scientific Experts in Tourism (AIEST) (p. 17-58). Calgary. 1987.

SACHS, Ignacy. Caminhos para o Desenvolvimento Sustentável. Rio de Janeiro: Garamond, 2004.

SAVAGE, G. T., Nix, T. W., Whitehead, C. J., Blair, J. D. Strategies for assessing and managing organizational stakeholders. Academy of Management Executive, 5(2), 1991, p. 61-75.

SOUSA, Almir Ferreira de; ALMEIDA, Ricardo Jose. O Valor da Empresa e a influência dos Stakeholders. São Paulo: Saraiva, 2006.

UNEP, Towards a Green Economy: Path ways to Sustainable Development and Poverty Eradication. 2011. Disponível em <www.unep.org/greeneconomy> Acesso em: 19 nov. 2014.

\section{Recebido em: 03/12/2015 (1 ${ }^{\text {a }}$ versão $)$ 13/06/2016 ( $2^{\text {a }}$ versão)}

Aprovado em: 21/07/2016 